Comparative Analysis of Trauma Outcomes between Rural and Urban Areas in Korea

Sung Woo Lee, MD; Chul Kyu Moon, MD; Jeon Min Jeon, $M D$; Sung Hyuk Choi, MD; Yun Sik Hong, MD

Department of Emergency Medicine, Korea University, College of Medicine, Seoul, KOREA

As the productive activities in the modern Korean society, have increased vastly following industrialization and urbanization, the resulting high mobility of people and goods have caused a sharp increase in the number of accidents that occur in the work places as well as by traffic accidents. In particular, deaths caused by injuries generally are concentrated in the economically active young people, producing incalculable losses to the society and nation as a whole.

Advanced nations with superior medical care systems have succeeded in reducing the incidence of such deaths by operating trauma centers. Especially noteworthy is the case of the United States where such specialized trauma centers have contributed greatly to reducing deaths from injuries in the non-urban areas with less access to medical facilities.

At present, Korea has no medical centers specializing in injuries. In the large cities, the injuries are being treated at third-tier medical facilities, while in the provinces, they are referred to small and medium-sized hospitals that constitute the second-tier medical facilities. Currently in Korea, the trauma patients are treated at general hospitals that consist of 726 second-tier medical facilities and 40 thirdtier medical centers nationwide. The second-tier medical facilities, which tend to take the most responsibility for the treatment of trauma generally are deficient in medical staff and facilities (including operating and intensive care facilities ). Despite such deficiencies and limitations, no regulations exist regarding treatment or transportation of trauma patients.

This article reports the outcome of a comparative analysis of the results of trauma treatments among different types of medical facilities based on objective data, with the hope that such study would facilitate a comparison with the treatment systems of advanced countries, and thereby contribute to a precise formulation of problems that must be addressed in this area.

Keywords: accidents; hospitals; industrial; industrialization; Korea; outcomes; rural; systems; trauma; TRISS; urban

E-mail: yunshong@unitel.co.kr

\section{Cooperation Between Civilians and Military During Disasters}

Dr. Beatriz Del Castillo 